\title{
Middle Eastern intestinal lymphoma: a morphological and immunohistochemical study
}

\author{
P ISAACSON*, HS AL-DEWACHI + , DY MASON $\ddagger$ \\ From the *Department of Pathology, Southampton University Medical School, Southampton, SO9 $4 X Y$, the \\ †Department of Pathology, Mosul University College of Medicine, Iraq, and the $¥ N u f f i e l d$ Department of \\ Pathology, John Radcliffe Hospital, Headington, Oxford, OX3 9DU
}

SUMmARY A total of 31 cases of Middle Eastern gastrointestinal lymphoma (from Mosul, Iraq) has been analysed by conventional histology, and also by immunoperoxidase staining of trypsinised paraffin sections with anti-Ig and anti-J chain antisera. Histologically these neoplasms fell into three categories: undifferentiated lymphoma of Burkitt type ( 8 cases); follicle centre cell (FCC) lymphoma, resembling European lymphomas of FCC origin (15 cases); and Mediterranean lymphoma (MTL) (3 cases). Immunohistological staining of the FCC neoplasms showed that these tumours resembled their European counterpart in that cytoplasmic Ig and $\mathbf{J}$ chain could be demonstrated in a proportion of cases. However there was a striking difference in that alpha chain alone, light chain alone, or $\mathbf{J}$ chain alone were detected in several cases (in contrast to the predominance of mu plus kappa or lambda light chains found in European cases). Furthermore, prominent intracellular inclusions of alpha chains were found in two cases. Staining of the MTL cases revealed that the characteristic mucosal plasma cell infiltrate was positive for alpha chain (weakly) and J chain (strongly) but that the invasive cells which morphologically resembled FCCs were negative for both constituents.

It is argued that these three histological categories constitute the major types of gastrointestinal lymphoma in the Middle East; and that in MTL the invasive lymphoma is of FCC type.

Continuing interest in primary gastrointestinal lymphoma as it occurs in the Middle East is occasioned by its remarkably high incidence in that area and by the fact that a high proportion of the cases demonstrate unusual specific clinicopathological features which have been characterised by the term Mediterranean lymphoma (MTL). An as yet unknown proportion of cases of MTL are associated with the presence in the serum or duodenal juice of an abnormal alpha heavy chain and the term alpha chain disease (ACD) has been used for these cases. ${ }^{1}$

The explanation of the high incidence of gastrointestinal lymphoma in Middle Eastern countries and the nature and interrelation of MTL and ACD continue to be poorly understood. Among the reasons for this is a lack of consensus regarding the histopathology of these tumours despite numerous publications from both the Middle East and elsewhere. $^{2-6}$ Changing concepts in lymphoma classification and poor tissue preservation in many

Accepted for publication 6 December 1982 of the cases have combined to give a confusing picture of Middle Eastern gastrointestinal lymphoma. In this study we report an analysis of 31 cases. We have made a histological diagnosis only from sections in which the tissue was well preserved and have complemented subjective observations with immunohistochemical techniques. Our results suggest that gastrointestinal lymphoma in the Middle East falls into three principal categories; undifferentiated (Burkitt-like) lymphoma, lymphoma of follicle centre cell (FCC) origin and MTL. Furthermore, the patterns of cytoplasmic Ig and $\mathrm{J}$ chain expression appear to differ in several respects from those commonly encountered in European non-Hodgkin's lymphoma.

\section{Material and methods}

The study was based on 31 cases of intestinal lymphoma from the files of Department of Pathology, Mosul Medical College, Iraq, which provides a diagnostic histopathology service for all teaching hospitals in Mosul and Nineveh provinces. Standard $5 \mu \mathrm{m}$ 


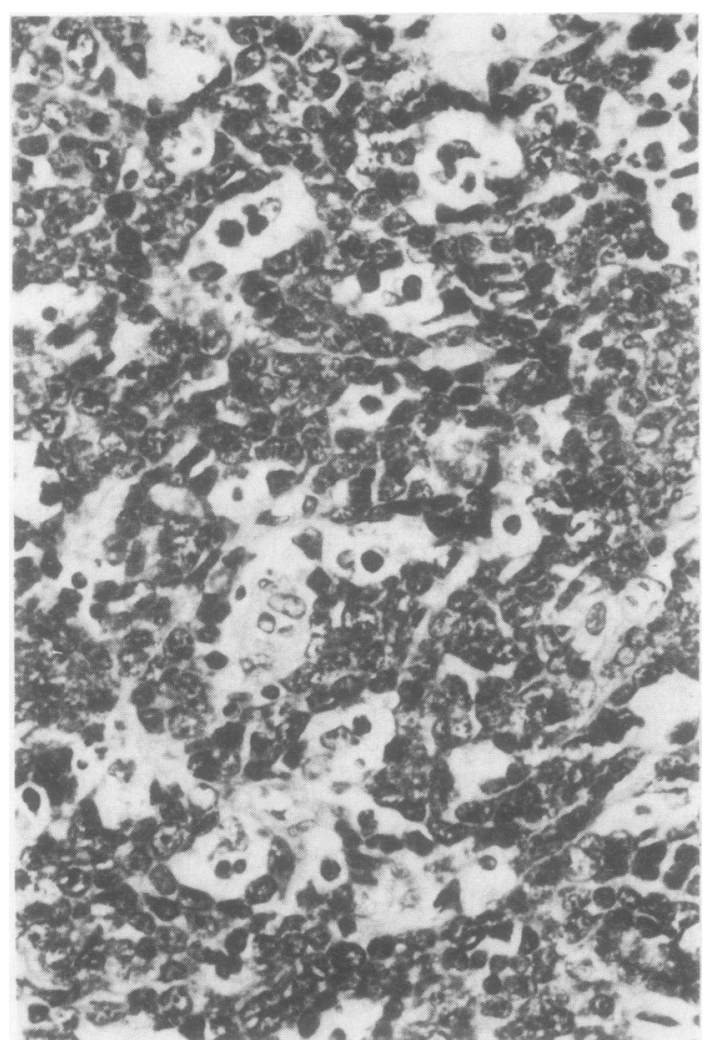

Fig. 1 Undifferentiated (Burkitt-like) lymphoma showing a monomorphic population of cells with darkly staining nuclei and scattered phagocytic histiocytes. Haematoxylin and eosin $\times 400$.

sections were prepared from the available paraffin blocks of these cases and were stained with haematoxylin and eosin. The tumours were then categorised according to the scheme described below and correlations made between the types of lymphoma, the age and sex of the patients, and the site of intestinal involvement. Sections from all cases were stained by the immunoperoxidase technique following trypsin digestion for $\alpha, \gamma, \& \mu$ immunoglobulin (Ig) heavy chains, $\kappa \& \lambda$ light chains and $J$ chain.* Details of the methods used have been previously described. ${ }^{78}$ To establish the optimum time necessary for trypsin digestion increasing digestion times and, if necessary, increasing concentrations of trypsin were used followed by staining with antisera against $\kappa \& \lambda$ chains. When optimum staining of reactive plasma cells was thus obtained additional sections were then stained for the antigens listed

\footnotetext{
*Antisera to human Ig components were purchased from Dako Immunoglobulins a/s Denmark. Antihuman J chain was purchased from Nordic Immunological Laboratories, Holland.
}

above. The titre of antiserum to $\alpha$ heavy chain was decreased tenfold (from $1 / 1000$ to $1 / 100$ ) when 은 staining those cases characterised morphologically as MTL.

\section{Results}

HISTOLOGICAL CATEGORIES

Eight cases were classified as undifferentiated lym- ֻ phoma (Burkitt-like) (Fig. 1). These were character- $\vec{\circ}$ ised by a monomorphic population of moderately large cells with little cytoplasm and nuclei containing $\vec{\omega}$ dark finely dispersed chromatin with 1-4 nucleoli. Scattered amongst these were phagocytic histiocytes with foamy cytoplasm giving the characteristic $\omega$ "starry sky" appearance.

Follicular centre cell (FCC) lymphoma was the diagnosis in 15 cases (Fig. 2). No attempt was made to subdivide these tumours which consisted of a var- 음 ying number of small centrocytes (small cleaved cells), large centrocytes (large cleaved cells) and $\bar{s}$ centroblasts (large non-cleaved cells). The irregular nuclear outlines of the FCCs allowed these tumours $\overrightarrow{0}$ to be clearly distinguished from the undifferentiated group. Plasmacytic differentiation was not a feature. In many cases much of the tissue was poorly fixed and the FCC nature of the tumour was not discernible (Fig. 3), the shrunken cells resembling small lymphocytes or plasmacytoid cells.

There were three cases of MTL (Fig. 4). In two of $\stackrel{\square}{\nexists}$ these the characteristic diffuse plasmacytic infiltra- $\frac{O}{3}$ tion was present both in adjacent small bowel mucosa and within the tumour. The sheets of plasma cells gave way to variably sized foci composed almost exclusively of cells morphologically identical with FCCs, while in some areas the two distinct cell $\bar{c}$ types were intimately mixed. In one case bizarre multinucleated cells were present amongst the $\delta$ FCCs. In the third case, available material consisted $₹$ of a mucosal biopsy which showed the characteristic 옹 plasma cell infiltrate of MTL and a lymph node $>$ which showed diffuse replacement by a mixture of follicle centre cells and mature plasma cells.

In five cases no confident morphological diagnosis o could be made. In four of these poor fixation was $N$ responsible, while in one the tumour consisted of ${ }_{\omega}^{N}$ bizarre large monomorphic cells with abundant 0 cytoplasm and prominent nuclei. This case could not 0 be placed into an established category of lymphoma $\underset{\mathbb{\Phi}}{\overparen{D}}$ and the possibility that it was an anaplastic tumour $\stackrel{\mathcal{?}}{?}$ of non-lymphoid origin could not be excluded.

\section{CLINICAL FEATURES (TABLES $1,2,3$ )}

Males outnumbered females by almost $2: 1$ in this $\frac{\stackrel{?}{\mathbb{Q}}}{\Omega}$ series but there were no significant sex differences 0 between the individual histological categories. The 


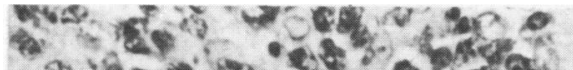

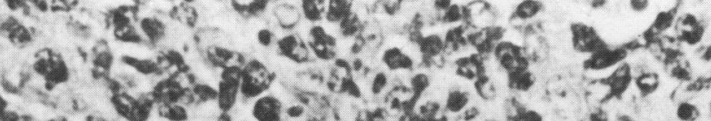

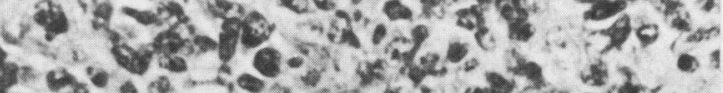

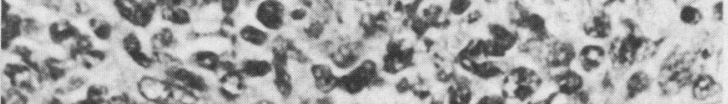

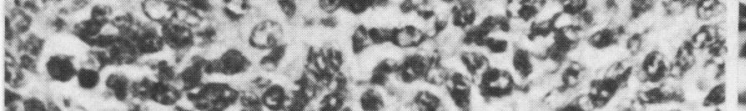
Sat -

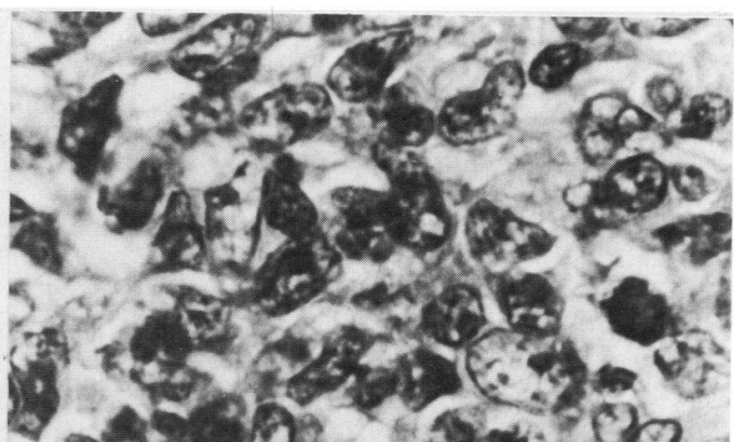

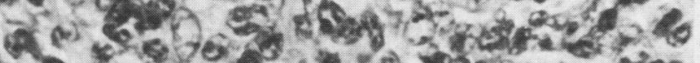

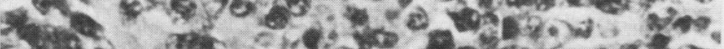

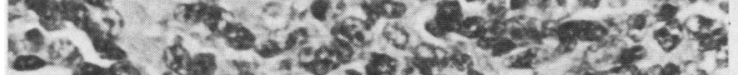

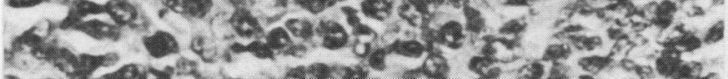

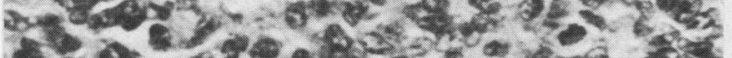

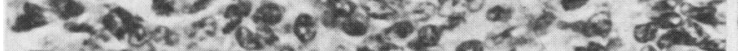
\&5 a

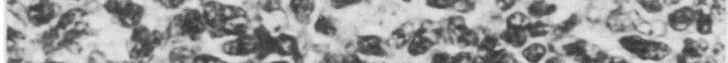

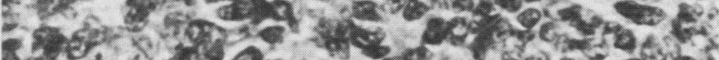

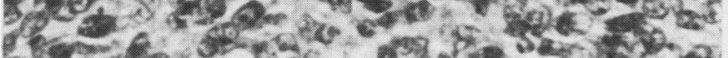
560.

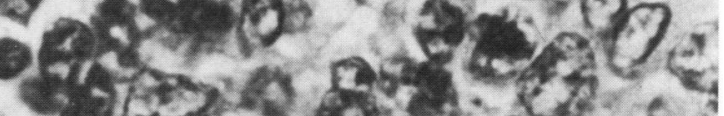

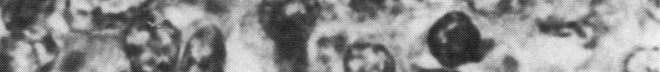

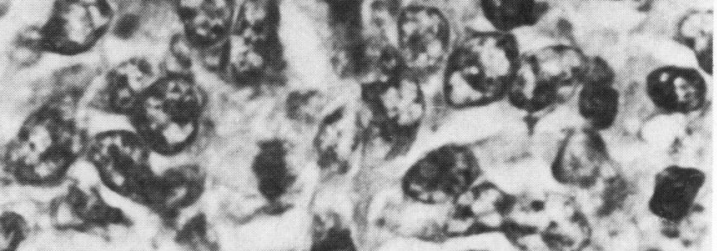

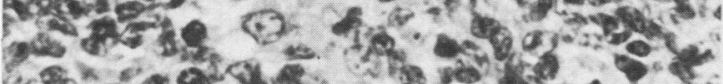

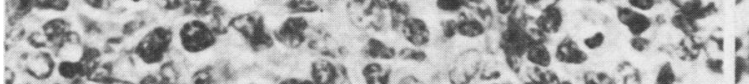

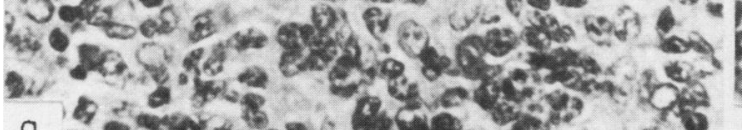

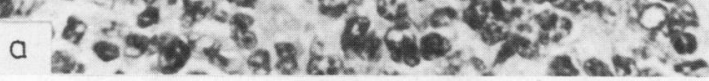

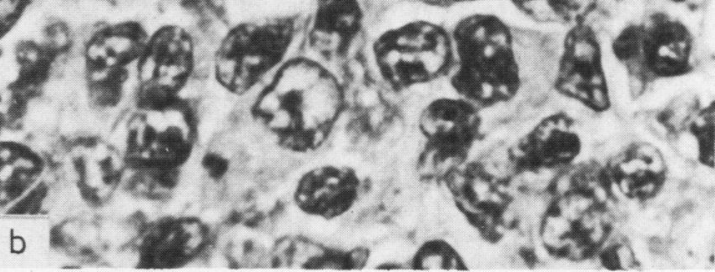

Fig. 2 Follicular centre cell (FCC) lymphoma showing a mixture of cells with irregular nuclear outlines and larger cells with clear, more rounded nuclei and prominent nucleoli, some of which are apposed to the nuclear membrane.

Haematoxylin and eosin (a) $\times 400 ;(b) \times 1000$.

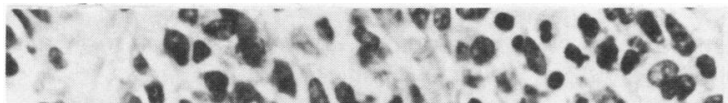
400909

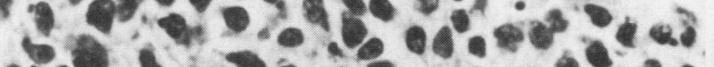

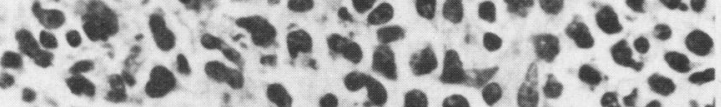

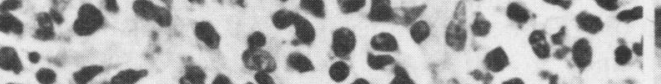

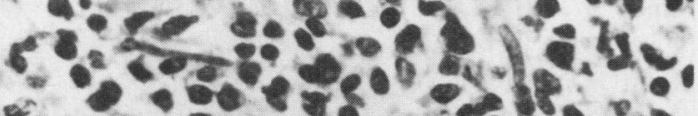
- ${ }^{2}$.

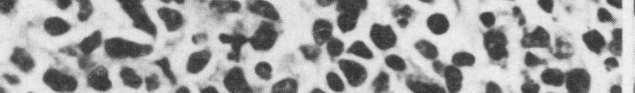

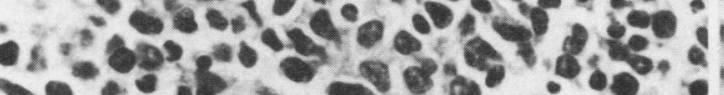

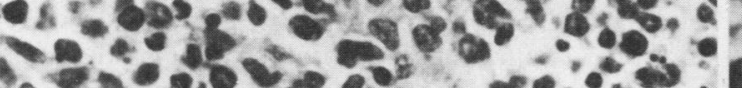

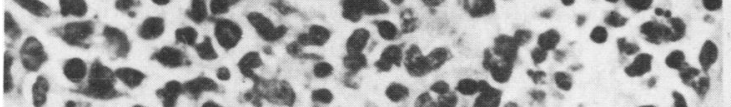

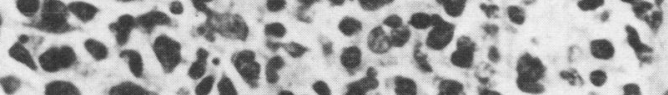

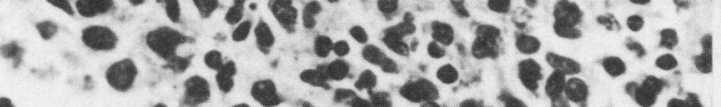

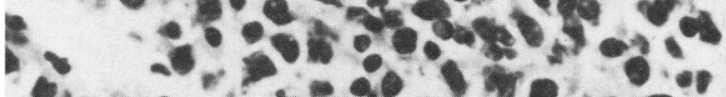
r. ne se 90

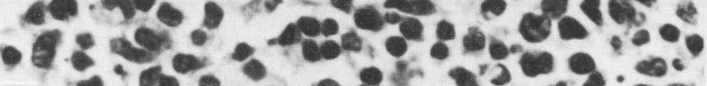

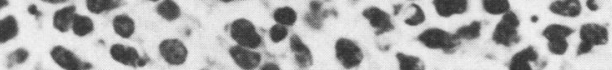
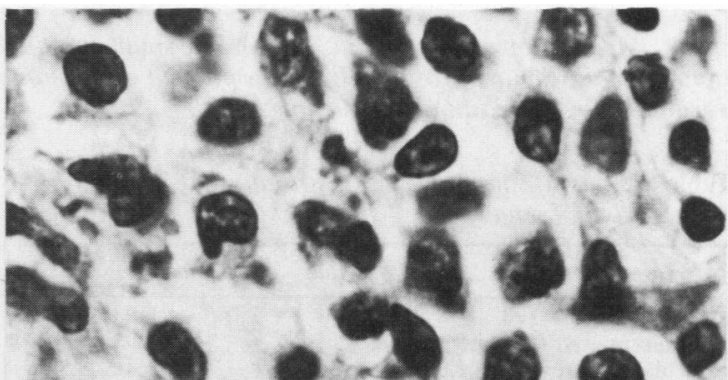

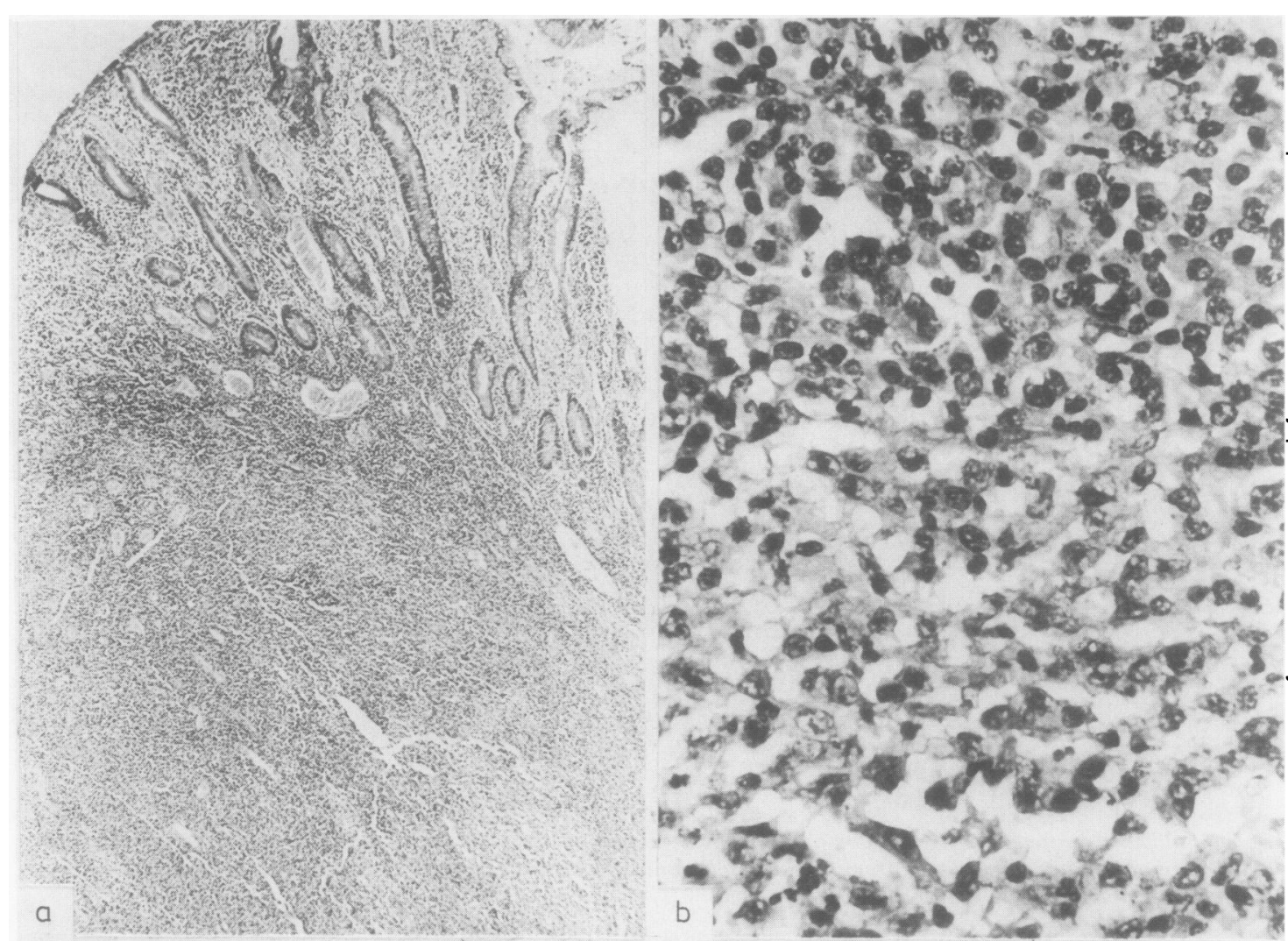

Fig. 4 Mediterranean lymphoma (MTL). The small intestinal mucosa adjacent to the tumour has lost its villi and shows an infiltrate in the lamina propria. High power detail of this tumour (right) shows distinct plasma cell and FCC areas.

Haematoxylin and eosin (a) $\times 40 ;(b) \times 400$.

Table 1 Classification and sex distribution of 31 cases of intestinal lymphoma

\begin{tabular}{lccc}
\hline & Male & Female & Total \\
\hline All cases & 20 & 11 & 31 \\
Undifferentiated (Burkitt-like) & 5 & 3 & 8 \\
Follicular Centre Cell (FCC) & 8 & 7 & 15 \\
Mediterranean Lymphoma (MTL) & 2 & 1 & 3 \\
Unclassified & 5 & - & 5 \\
\hline
\end{tabular}

peak age incidence was in the third decade but the majority of undifferentiated lymphomas occurred in children. There was relative sparing of the midportion of the small intestine with the majority of undifferentiated lymphomas occurring distally, while cases of MTL involved the proximal smallo bowel. Cases of FCC lymphoma were equally divided between the proximal and distal small intes-ô tine.

IMMUNOCHEMISTRY (TABLE 4) of the cases of undifferentiated (Burkitt-like) lym-n phoma. Immunoperoxidase staining showed $a^{-}$ monotypic Ig pattern in seven cases of FCC lym- $N$ phoma (Fig. 5). Of these four contained FCCs stain- $-\tilde{\omega}$ ing for $\alpha$ chain alone with no light chains demons-

Table 2 Ages (yr) of 31 patients with intestinal lymphoma-

\begin{tabular}{|c|c|c|c|c|c|c|c|}
\hline & $0-10$ & $11-20$ & $21-30$ & $31-40$ & $41-50$ & $51-60$ & $>60$ \\
\hline $\begin{array}{l}\text { All cases } \\
\text { Ulndifferentiated }\end{array}$ & $\begin{array}{l}6 \\
5\end{array}$ & 4 & 11 & 6 & $\begin{array}{l}3 \\
1\end{array}$ & 二 & 1 \\
\hline $\begin{array}{l}\text { Undifferentiated } \\
\text { FCC }\end{array}$ & 1 & 1 & $\overline{7}$ & 4 & $\begin{array}{l}1 \\
1\end{array}$ & - & $\overline{1}$ \\
\hline $\begin{array}{l}\text { MTL } \\
\text { Unclassified }\end{array}$ & - & $\begin{array}{l}1 \\
1\end{array}$ & $\begin{array}{l}1 \\
3\end{array}$ & $\overline{1}$ & 1 & 二 & - \\
\hline
\end{tabular}


Table 3 Site of tumour in 31 cases of intestinal lymphoma

\begin{tabular}{lrlrl}
\hline & Prox & Mid & Distal & $\begin{array}{l}\text { Not } \\
\text { specified }\end{array}$ \\
\hline All cases & 10 & 4 & 13 & 4 \\
Undifferentiated & 2 & 2 & 4 & $\frac{2}{2}$ \\
FCC & 5 & $\frac{1}{8}$ & $-\frac{1}{2}$ \\
MTL & 2 & 1 & 1 & $\frac{1}{\text { Unclassified }}$ \\
\hline
\end{tabular}

Prox $=$ duodenum $\&$ jejunum.

Mid = proximal ileum.

Distal = ileum \& ileocaecal.

NS $=$ not specified.
Table 4 Cases showing positive IP staining in 31 cases of gastrointestinal lymphoma

\begin{tabular}{|c|c|c|c|c|c|c|}
\hline Type & $\alpha$ & $\gamma$ & $\boldsymbol{\mu}$ & $\kappa$ & $\lambda$ & $J$ \\
\hline FCC & + & - & - & - & - & + \\
\hline FCC & + & - & - & - & - & + \\
\hline FCC & $+^{*}$ & - & - & - & - & - \\
\hline FCC & $+^{*}$ & - & - & - & - & + \\
\hline FCC & - & - & - & + & - & + \\
\hline FCC & - & - & - & - & + & + \\
\hline FCC & - & - & - & - & - & + \\
\hline MTL & + plasma cells $\dagger$ & - & - & - & - & + plasma cells \\
\hline MTL & + plasma cells $\dagger$ & - & - & - & - & + plasma cells \\
\hline MTL & + plasma cells $\dagger$ & - & - & - & - & + plasma cell \\
\hline
\end{tabular}

${ }^{*}$ Present as inclusion bodies.

†Anti $\alpha$ used at $1 / 100$.

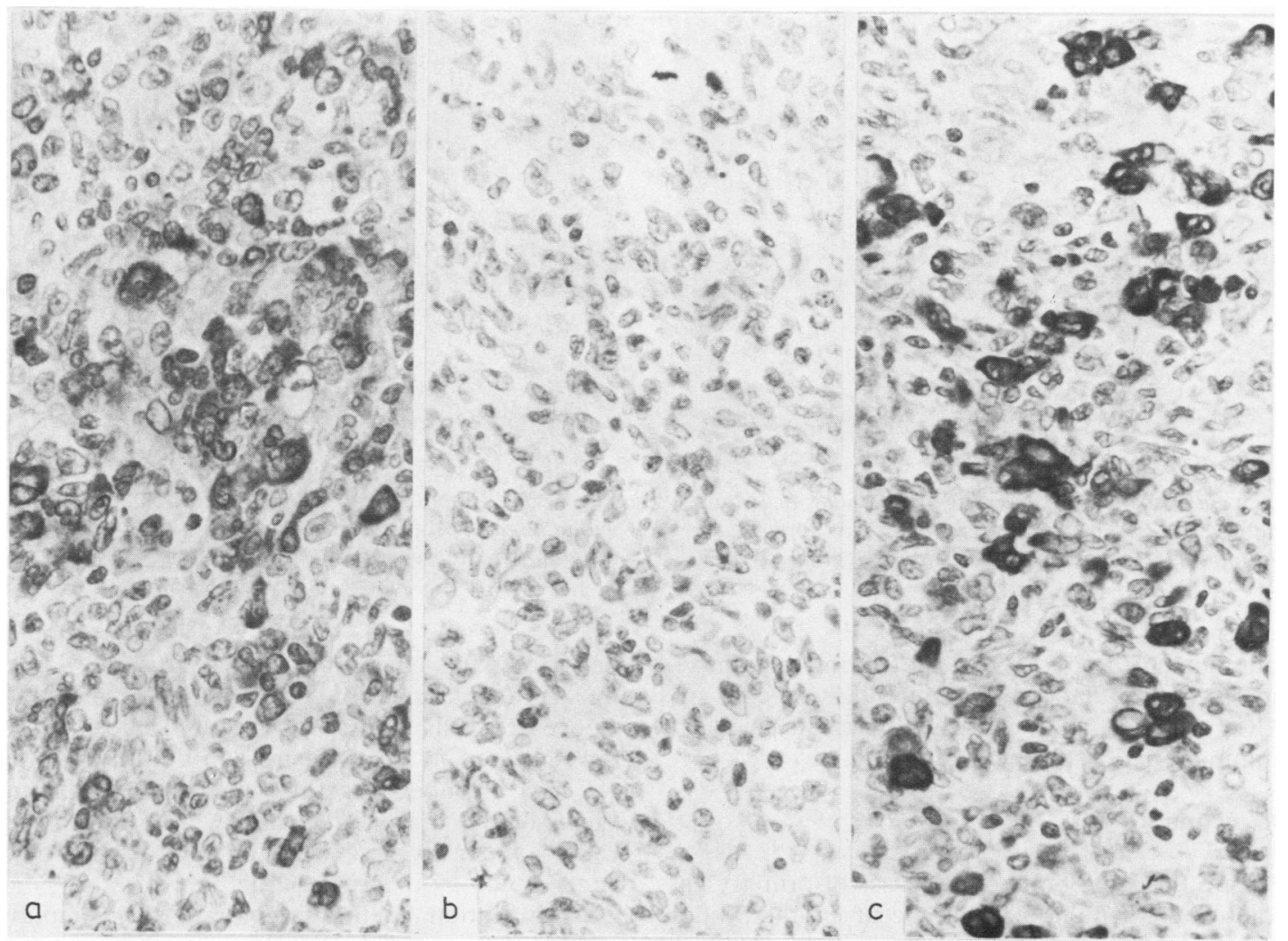

Fig. 5 FCC lymphoma stained for (a) $\kappa$ chain (b) $\lambda$ chain and (c) $\mathrm{J}$ chain. There is monotypic staining of Clg within FCCs which also contain $J$ chain. Immunoperoxidase $\times 400$.

trated. Three of these also stained for J chain. The $\alpha$ chain in two cases appeared as dark staining granular inclusions (Fig. 6). This positive $\alpha$ chain staining was obtained with the standard dilution of antiserum $(1 / 1000)$. In the remaining three cases no heavy chain could be demonstrated and tumour cells could be shown to contain respectively $\kappa$ and $J$ chains, $\lambda$ and $\mathrm{J}$ chains and $\mathrm{J}$ chain alone.
The plasma cells in the three cases of MTL stained strongly for $J$ chain (Fig. 7) and weakly for $\alpha$ chain with the standard dilution of anti- $\alpha$ antiserum but strongly when the dilution was increased tenfold (Fig. 8). However, the islands of FCCs and isolated FCCs in these cases consistently failed to stain, as did the large bizarre multinucleated cells in the single case in which they were present. In the lymph 


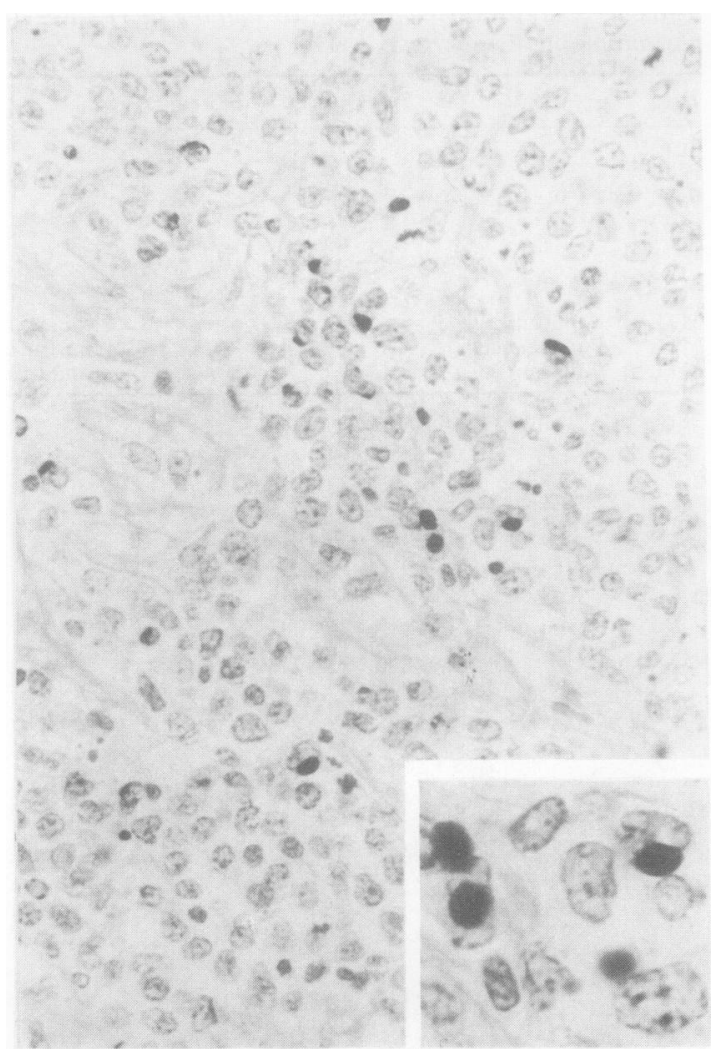

Fig. 6 FCC lymphoma showing intracellular inclusions staining for $\alpha$ chain. The antiserum has been applied at a dilution of 1/1000. Immunoperoxidase $\times 400$; inset $\times 1000$.

node involved by MTL the intimately mixed dimorphic population of mature plasma cells and FCCs could be clearly distinguished by immunoperoxidase staining (Fig. 9).

\section{Discussion}

Despite the large numbers of cases of primary gastrointestinal lymphoma that occur in the Middle East the histology of the tumours is a subject fraught with confusion. Part of the difficulty has arisen because of attempts to analyse tissue that is poorly preserved. As shown in this study the appearance of a tumour alters radically with poor fixation. Nuclei lose their characteristic shapes as a result of shrinkage and the cytoplasm becomes drawn out giving an impression of plasmacytic or plasmacytoid differentiation.

The interpretative difficulties arising from these technical artefacts are compounded by the current confusion which surrounds the classification of

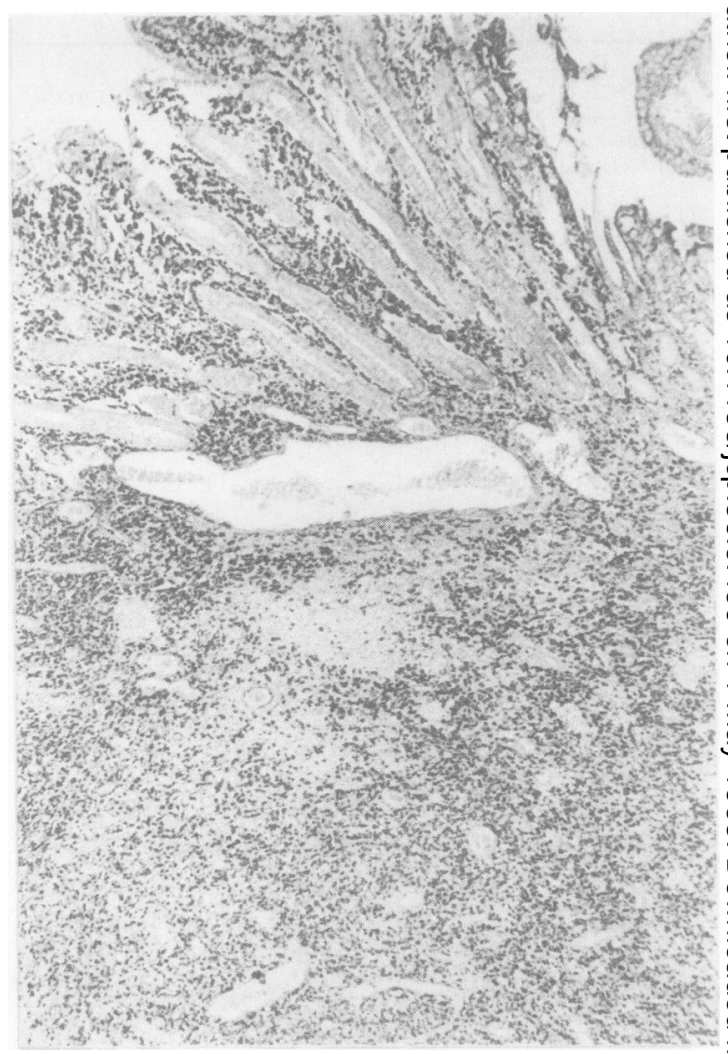

Fig. 7 MTL stained for J chain. There are negative foci within a sea of positively staining plasma cells. Immunoperoxidase $\times 40$.

non-Hodgkin's lymphoma. This confusion is largely음 due to the multitude of terms used to describe follicle centre cells (FCC) at various stages in theirô development. It is for this reason that in this study we have grouped together under the single title음 "FCC lymphoma" all the cases which, morphologi- $\rightarrow$ cally, appear to arise from B cells of the follicle? centres.

Classification of the samples in this simplified ${ }^{\circ}$ fashion makes it clear that there are three principal 0 types of Middle East gastrointestinal lymphoma. The first of these is an undifferentiated (Burkittlike) lymphoma, most commonly seen in children'o and closely resembling childhood intestinal lymphoma tumour seen in Western countries. ${ }^{10}$ The? second category is FCC lymphoma which is also wello recognised in the West; whilst the third type is? Mediterranean lymphoma, a neoplasm which, in contrast to the two previous categories, is rarely, if ever, seen in Western countries.

The division into three types is also evident when 


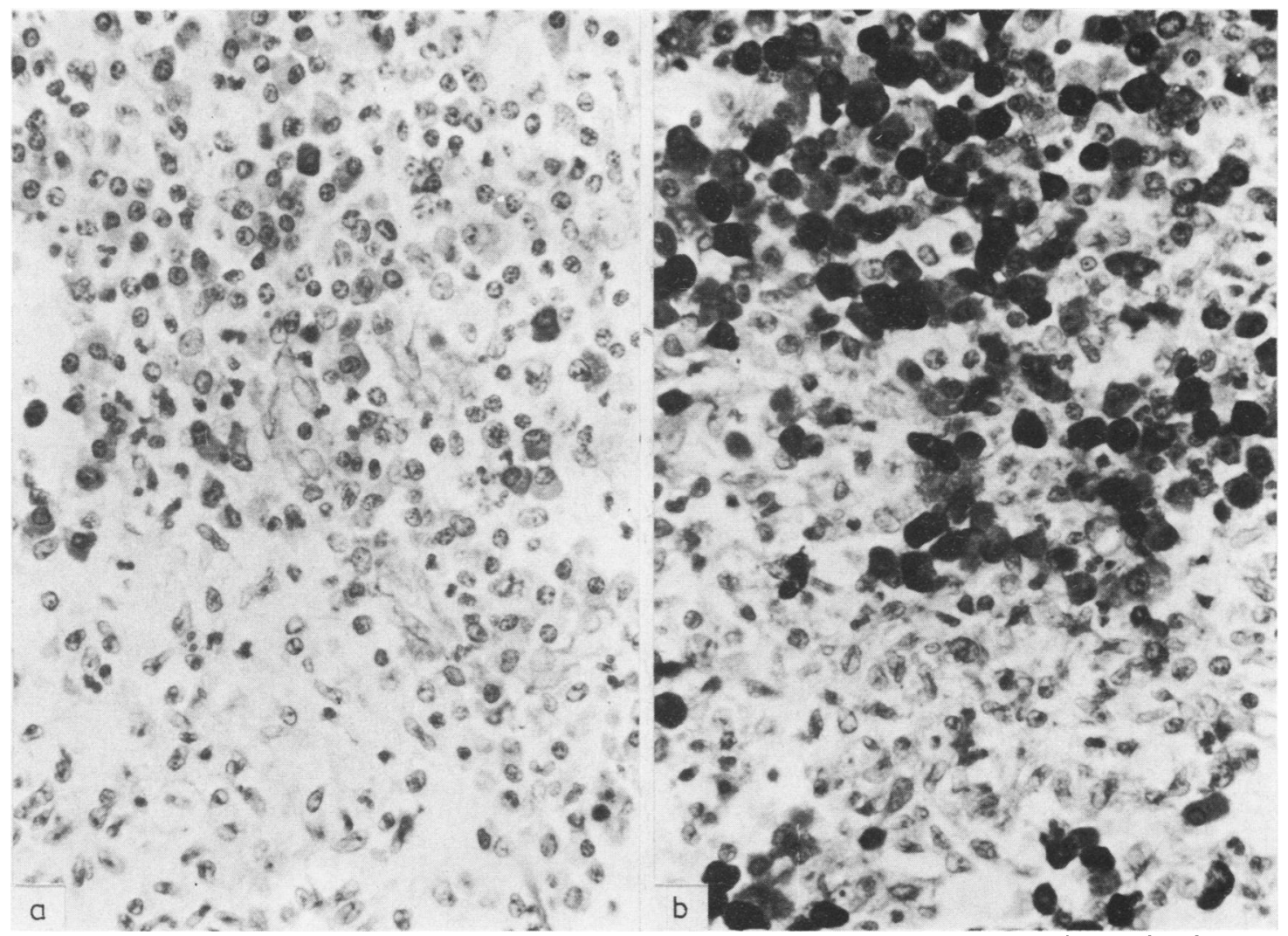

Fig. 8 Sequential sections of MTL stained with anti- $\alpha$-antiserum at (a) $1 / 1000(b)$ and $1 / 100$. The abnormal $\alpha$-chain in the plasma cells is best stained with the more concentrated antiserum (compare Fig. 6). Note the Ig-negative FCCs.

Immunoperoxidase $\times 400$.

other large series of gastrointestinal lymphoma reported from the Middle East are analysed. Both Selzer et al, ${ }^{5}$ who reported 43 cases from Israel, and $\mathrm{Al}$ Saleem and Zardawi ${ }^{6}$ who described 145 cases from Iraq recognised undifferentiated (Burkitt-like) lymphoma and MTL(ACD) as distinct groups within their series. With the exception of five plasmacytomas Al Saleem and Zardawi categorised the remainder of their cases as moderately or poorlydifferentiated diffuse lymphocytic lymphomas. Although none of these cases was illustrated, the use of this term, which is from the Rappaport classification, ${ }^{11}$ infers that the tumours were composed of FCCs. Selzer et al clearly illustrated FCC tumours and used a variety of terms from the BNL1 classification $^{12}$ to categorise a large group of their cases. These terms can be equated with those used by Lennert ${ }^{13}$ and Lukes and Collins ${ }^{14}$ to describe FCC lymphomas.

Extensive immunoperoxidase studies of nodal FCC lymphoma of European origin in the past have demonstrated intracytoplasmic Ig of a single light chain class (usually with associated $J$ chain) in up to $65 \%$ of cases. ${ }^{15-17}$ It should be emphasised that the Ig in such cases is present within FCCs and not plasma cells. The results of immunoperoxidase staining of FCC lymphomas in the present series paralleled these previous investigations, in that approximately $50 \%$ showed a monotypic staining pattern. The fact that this figure was somewhat lower than expected on the basis of previous experience may well reflect the fact that many of these cases appeared to have been fixed in formalin for long periods before embedding. This was inferred from the long trypsin digestion times required in order to obtain satisfactory staining. It is probable that this prolonged exposure to fixatives produced artefactually negative staining results in a number of cases.

Although the finding of monotypic cytoplasmic Ig in several of the FCC lymphomas in this series paralleled the pattern found in European FCC lym- 

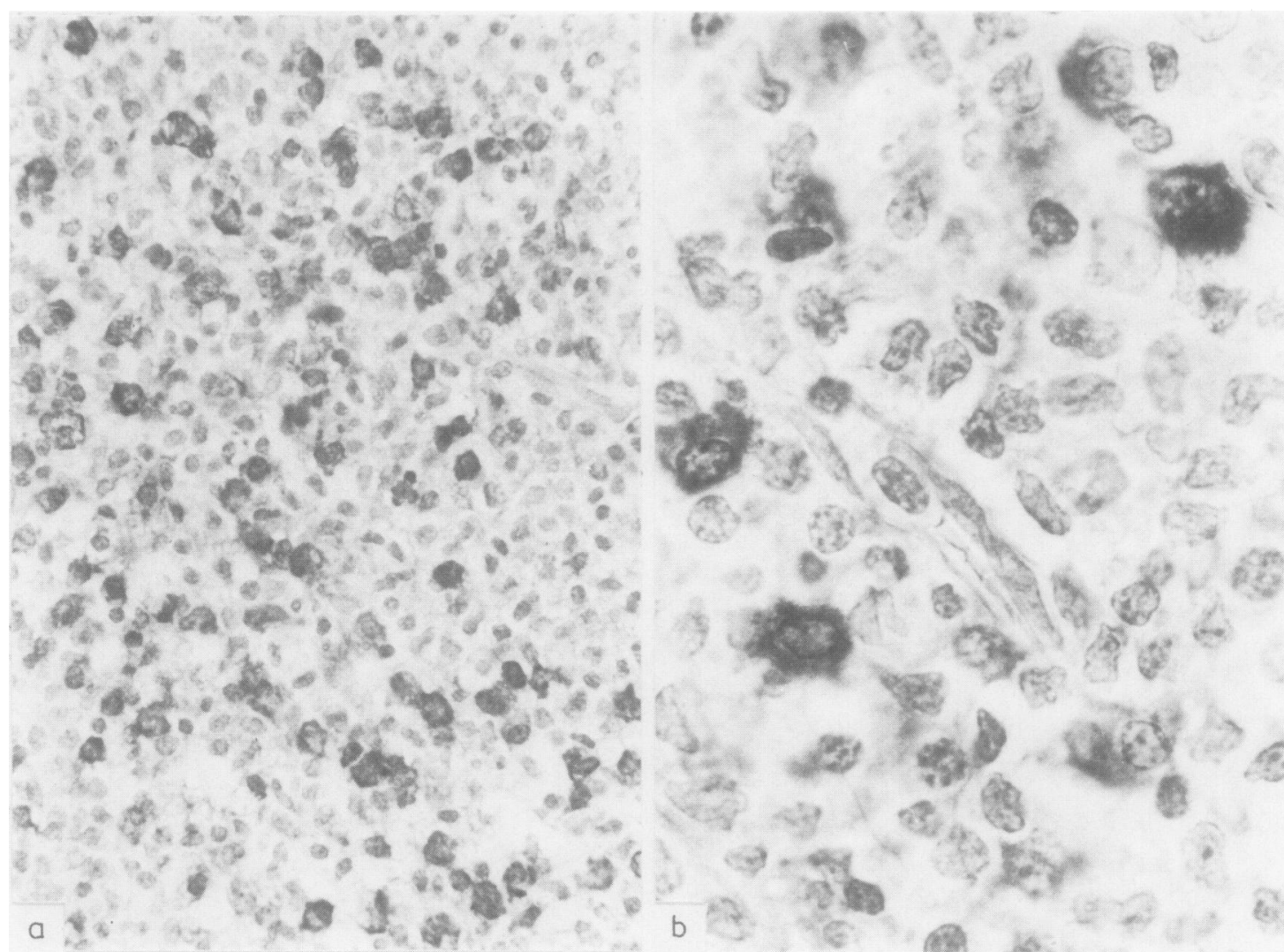

Fig. 9 Lymph node from a case of MTL stained for $\alpha$-chain. The mixture of Ig-positive and negative cells is clearly shown in the high power view to be plasma cells and FCCs. Immunoperoxidase $(a) \times 40,(b) \times 1000$.

phomas, there was a striking difference in the type of Ig present. The Middle East cases stained for alpha chain alone or light chain alone, whilst one case stained for $\mathbf{J}$ chain alone. In contrast European cases of FCC lymphoma almost always stained for mu chains accompanied by light chain, and we are not aware of any previous reports of FCC lymphomas staining for light chain or $\mathrm{J}$ chain alone. Furthermore, it is of interest that in two cases intracellular inclusion bodies consisting of alpha chains (unaccompanied by light chain or $\mathbf{J}$ chain) were prominent. Such pure alpha chain inclusions have not previously been described.

The explanation for the unusually high frequency of alpha chain-positive FCC lymphomas in this series is not apparent although it may reflect the fact that these cases were of gastrointestinal rather than nodal origin. It should be emphasised that there was no suggestion that the "alpha chain only" cases were in any way related to MTL. Not only did these tumours lack any histological resemblance to MTL, but in addition the non-malignant areas of mucosa, when present, were free of a plasmacytic infiltrate of용 the alpha chain disease type. Furthermore, it should 0 be noted that the alpha chain present in the FCC $\stackrel{3}{\text {. }}$. lymphoma cells stained strongly with normalo concentrations of anti-alpha chain antiserum. This $₹$ contrasts with the pattern in classical ACD, in which 을 the lamina propria infiltrate can only be stained satisfactorily by using high concentrations of anti-을. alpha chain antiserum.

Histologically the FCC tumours occurring in the ${ }^{\circ}$ Middle East are not obviously different from those $N$ that occur in the West. Almost all the tumours are diffuse and, as in the undifferentiated cases, there is no specific associated mucosal abnormality. The his-o tology of these cases varies considerably depending

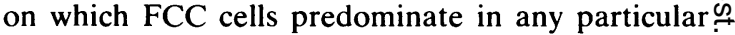
case but there is little point in subdividing these $\square$ tumours any further at this time, although this may be important when considering prognosis. Common aetiological factors if they exist are likely to apply to $\stackrel{\mathbb{Q}}{\otimes}$ all FCC tumours.

The high incidence of undifferentiated (Burkitt- 
like) lymphoma occurring in the Middle East is evident from many publications from that region. ${ }^{9}$ The cases occur most frequently but not exclusively, in children and there is no associated mucosal abnormality. Histologically, there is close resemblance to Burkitt's lymphoma but whether these cases are true examples of Burkitt's lymphoma awaits further immunological and viral studies.

In a previous study of five cases of primary gastrointestinal lymphoma from the Middle East a characteristic staining pattern was noted when mucosal biopsies from patients with ACD were stained for Ig using immunoperoxidase. ${ }^{18}$ In these cases the plasma cells stained monotypically for $\alpha$ chain but only when higher than usual concentrations of antiserum were used. The cells also stained very strongly for $\mathbf{J}$ chain. Well defined foci of negatively staining cells were observed and since they appeared to be invading and destroying crypts it was suggested that these cells represented the earliest stage of invasive lymphoma although no actual tumour tissue was available for study. It was also suggested on morphological criteria, that the invading cells were most likely of FCC origin.

The present study provides further illustration of the distinctive dual population of cells in MTL, with islands of Ig and $\mathbf{J}$ chain-negative FCCs being surrounded by mature $\alpha$ and $\mathrm{J}$ chain-positive plasma cells. In mesenteric lymph nodes, and to some extent in the intestinal tumours, these two types of cell are intimately mixed and the resulting impression may be that of plasmacytic differentiation in an FCC tumour. Immunoperoxidase staining for $\mathbf{J}$ chain or $\alpha$ chain clearly demonstrates the distinctive dual cell population of MTL, the FCC component of the disease having evolved against the background of the mature plasma cell infiltrate. This dual cell population in MTL has been commented on by others ${ }^{5}$ but its significance has not been understood. These appearances do not, in our view, support the usual explanation of the sequence of events in MTL which is that the tumour starts as a plasmacytoma and gradually "dedifferentiates" into a so-called immunoblastic sarcoma in much the same way as sometimes occurs in skeletal plasma cell myeloma. ${ }^{20}$ It seems more likely that the relation between FCCs and plasma cells in MTL is the same as that in the normal intestine. Here, following antigenic stimulation, FCCs migrate via intestinal lymphatics, mesenteric lymph nodes and the thoracic duct into the peripheral circulation whence they home to the lamina propria as plasma cells. ${ }^{19}$ The sharp distinction between FCCs and plasma cells in MTL is a parody of the normal anatomical relation between these two cell types in the intestinal lamina propria. MTL could thus be regarded as a tumour of lamina propria FCCs which, since they are still receptive to antigenic drive, results, in the early stages, in massive plasmacytosis of the intestinal lamina propria. The FCC element of the tumour is thus at first obscured but emerges in the later stages of the disease.

A further point raised by these immunohistochemical studies relates to the question as to whether MTL and ACD are synonymous. It has been suggested that those cases of MTL in which the abnormal $\alpha$ chain cannot be demonstrated in serum or duodenal juice are examples of non-secretory ACD. ${ }^{21}$ Until such time as immunohistochemical studies demonstrate other immunoglobulins in the plasma cells of MTL we would support this suggestion which helps to simplify the concept of MTL within the wider spectrum of intestinal lymphoma.

We are grateful to Rita-Elizabeth Woolston for skilful technical assistance and to Olive Huber for typing the manuscript. Part of this work was supported by a grant from the Leukaemia Research Fund (DYM).

\section{References}

${ }^{1}$ World Health Organisation. Alpha-chain disease and related small-intestinal lymphoma: a memorandum. Bull WHO 1976;54.

: Galian A, Lecestre M-J, Scotto J, Bognell C, Matuchansky C, Rambaud J-C. Pathological study of alpha-chain disease, with special emphasis on evolution. Cancer 1977;39:2081-101.

${ }^{3}$ Haghighi P, Kharazmi A, Gerami C, Haghshenass M, Abadi P, Omidi P, Mostafavi N. Primary upper small-intestinal lymphoma and alpha-chain disease. Report of 10 cases emphasising pathological aspects. Am J Surg Pathol 1978;2:147-57.

${ }^{4}$ Nassar VH, Salem PA, Shahid MJ, Alami SY, Balikian JB, Salem AA, Nasrallah SM. "Mediterranean abdominal lymphoma“ or immunoproliferative small intestinal disease. Part III. Pathological Aspects. Cancer 1978;41:1340-54.

${ }^{s}$ Selzer G, Sherman G, Callihan TR, Schwartz Y. Primary small intestinal lymphomas and $\alpha$-heavy-chain disease. A study of 43 cases from a pathology department in Israel. Isr J Med Sci 1979;15:111-23.

- Al-Saleem T, Zardawi IM. Primary lymphomas of the small intestine in Iraq: a pathological study of 145 cases. Histopathology 1979;3:89-106.

${ }^{7}$ Mepham BL, Frater W, Mitchell BS. The use of proleolytic enzymes to improve Ig staining by the PAP technique. $J$ Histochem 1979;11:345-57.

${ }^{8}$ Issacson P. Immunochemical demonstration of $\mathrm{J}$ chain: a marker of B-cell malignancy.J Clin Pathol 1979;32:802-7.

9 Al-Attar A, Al-Mondhiry H, Al-Bahrani Z, Al-Saleem T. Burkitt's lymphoma in Iraq. Clinical and pathological study of forty-seven patients. Int J Cancer 1979;23:14-7.

1" Crist WM, Kelly DR, Ragab AH, Roper M, Dearth JC, Castleberry RP, Flint A. Predictive ability of Lukes-Collins classification for immunological phenotypes of childhood non-Hodgkin lymphoma. An institutional series and literature review. Cancer 1981;48:2070-5.

"Rappaport H. Tumors of the hematopoietic system. Atlas of tumor pathology Section 3, Fascicle 8. Washington DC: Armed Forces Institute of Pathology, 1966. 
${ }^{12}$ Henry K, Bennett MH, Farrer-Brown G. Classification of the non-Hodgkin's lymphoma. In: Anthony PP, Woolf N, eds. Recent advances in histopathology. London: ChurchillLivingstone, 1978.

${ }^{13}$ Lennert K, Mohri N, Stein H, Kaiserling E, Muller-Hermelink HK. Malignant lymphomas other than Hodgkin's disease. Berlin: Springer-Verlag, 1978.

${ }^{14}$ Lukes RJ, Collins RD. New approaches to the classification of lymphoma. Symposium on non-Hodgkin's lymphoma. $\mathrm{Br} J$ Cancer 1975;31 suppl 2:1-28.

15 Mason DY, Bell JI, Christensson B, Biberfeld P. An immunohistological study of human lymphoma. Clin Exp Immunol 1980;40:235-48.

${ }^{16}$ Isaacson P, Wright DH, Judd MA, Jones DB, Payne SV. The nature of the immunoglobulin-containing cells in malignant lymphoma. An immunoperoxidase study. J Histochem Cytochem 1980;28:761-70.

${ }^{17}$ Landaas TO, Godal T, Marton PF, Kvaløy S, Langholm R, Lindom T, Jørgensen OG, Høst H. Cell-associated immunoglobulin in human non-Hodgkin lymphomas. Acta Pathol Microbiol Scand $[A]$ 1981;89:91-10.

18 Isaacson P. Middle East lymphoma and $\alpha$-chain disease. An immunohistochemical study. Am J Surg Pathol 1979;3:431-응 41.

19 Parrot DMV: The gut as a lymphoid origin. Clin Gastroentero 1976;5:211-28.

${ }^{20}$ Rambaud JC, Galian A, Matuchansky S, Danon F, Preu $\bar{Q}$ 'Homme JL, Brouet JC, Seligmann M. Natural history ố $\alpha$-chain disease and the so-called Mediterranean lymphoma $\bar{n}$ Mathe G, Seligmann M, Tubiana M, eds. Recent results ir cancer research. Lymphoid neoplasias I; classification, categor ization. Natural History. Berlin: Springer-Verlag, 1978.

${ }^{21}$ Rambaud JC, Modigliana R, Nguyen Phuoc BK: Non-secretory alpha-chain disease in intestinal lymphoma. $N$ Engl $J$ Med 1980;303:53.

Requests for reprints to: Professor P Isaacson, Department? of Morbid Anatomy, School of Medicine, University Col- $\omega$ lege London, University Street, London WC1E 6JJ, Eng-i land. 